\title{
Bone marrow-derived dendritic cells under influence of experimental breast cancer and physical activity
}

\author{
DOUGLAS R. ABDALLA ${ }^{1}$, BRUNO B. M. GOMES ${ }^{1}$, EDDIE F. C. MURTA ${ }^{2}$ and MÁRCIAA. MICHELIN ${ }^{3}$ \\ ${ }^{1}$ Oncology Research Institute; Disciplines of ${ }^{2}$ Gynecology and Obstetrics and ${ }^{3}$ Immunology, \\ Oncology Research Institute, Federal University of the Triângulo Mineiro, Uberaba, MG 38025-180, Brazil
}

Received May 20, 2016; Accepted July 28, 2016

DOI: $10.3892 / 01.2017 .5589$

\begin{abstract}
Immune cells are required in the immune response against tumours, although sometimes without success. The present study aimed to investigate dendritic cell (DC) maturation in animals with induced immunosuppression that were subjected to physical activity (PA). Immunosuppression was induced using 7,12-dimethyl-benzanthracene (DMBA). A total of $56 \mathrm{Balb} / \mathrm{c}$ mice were divided into four groups, including the control group, non-DMBA administered/PA group (GII), DMBA administered/non-PA group (GIII) and the DMBA administered/PA group (GIV). Bone marrow was removed from the leg bones following sacrifice. Bone marrow-derived DCs were stimulated to differentiate by granulocyte-macrophage colony-stimulating factor, interleukin (IL)-4 and tumour necrosis factor- $\alpha$, after which the phenotype was assessed by flow cytometry and the cytokine profile was assessed using ELISAs. PA significantly increased the percentage of DCs in GII (55.38 $2.63 \%)$ and GIV (50.1 $43.1 \%)$ mice, as compared with GI $(34.61 \pm 1.28 \%)$ and GIII $(36.25 \pm 1.85 \%)$ mice $(\mathrm{P}<0.05)$. In addition, GIV mice showed a significantly higher level of cluster of differentiation (CD) $80^{+} / \mathrm{CD}^{2} 6^{+} \mathrm{DCs}(76.38 \pm 6.31 \%)$, as compared with GI $(54.03 \pm 6.52 \%)$ and GIII $(52.07 \pm 5.74 \%)$ mice $(\mathrm{P}<0.05)$. Furthermore, GIV mice showed a significantly higher level of $\mathrm{CD} 80^{+} /$major histocompatibility complex class II double labelling $(\mathrm{P}<0.05)$, as compared with GIV $(95.35 \pm 1.22 \%)$ and GIII $(76.15 \pm 5.53 \%)$ mice. The expression of interferon- $\gamma$ was significantly increased in GIV mice [5.89 (5.2-7.12)], as compared with GIII mice [2.75 (1.33-4.4)] $(\mathrm{P}<0.05)$. Similarly, the expression of IL-12 was markedly increased in GIV mice [1.27 (0.26-2.57)] compared with GIII mice [0.73 (0.44-1.47)], although the difference was not significant $(\mathrm{P}=0.063)$. The results of the present study suggested that
\end{abstract}

Correspondence to: Professor Márcia A. Michelin, Discipline of Immunology, Oncology Research Institute, Federal University of the Triângulo Mineiro, 1940 Avenida Guilherme Ferreira, Uberaba, MG 38025-180, Brazil

E-mail: michelinimuno@icbn.uftm.edu.br

Key words: immune response, immunosuppression, immunotherapy, physical activity, dendritic cells
PA was able to promote the maturation of DCs and their secretion of anti-tumour cytokines. Therefore, PA may emerge as a tool in immunotherapy.

\section{Introduction}

Dendritic cells (DCs) are the most potent antigen-presenting cells; thus, investigation of these cells is crucial to elucidate the functions of the immune system. The maturation state of DCs has a pivotal role in their activity, since it influences their ability to uptake, process and present antigens, as well as their migration to lymph nodes that drain tumour sites and their stimulation of a potent T-cell-mediated anti-tumour response. Immature DCs are more efficient at capturing and processing antigens than mature DCs. Furthermore, immature DCs have been shown to migrate more effectively to lymph nodes where they undergo maturation and upregulate the expression of costimulatory and major histocompatibility complex (MHC) molecules, resulting in a T-cell response (1-4).

Tumour escape from the immune response may be due to the decreased or absent expression of MHC molecules and/or costimulatory molecules. In addition, the secretion of immunosuppressive factors by tumours, such as transforming growth factor- $\beta$ (TGF- $\beta$ ), interleukin (IL)-10, vascular endothelial growth factor and IL-4, as well as prostaglandin E2, nitric oxide, soluble IL-2 receptors, complement inhibitors, proteases, gangliosides, hexosamines, $\alpha$-fetoprotein, fibronectin and phosphatidylserine, may inhibit the anti-tumour immune response (5-8).

Previous studies have suggested that there is an important association between physical activity (PA) and the immune system (9-12), although the underlying mechanisms are unknown. LaVoy et al (9) demonstrated that a single session of dynamic exercise could increase the differentiation of monocyte-derived DCs in healthy individuals. Furthermore, the practice of certain activities was hypothesized to contribute to the prevention of, or serve as an adjunct to the treatment of, diseases such as cancer, type 2 diabetes, arthritis and other chronic diseases (10-12). Chronic diseases represent the leading causes of mortality worldwide (13), and the clinical use of non-pharmacological interventions for immune activation may improve the quality of life of patients with chronic diseases.

With respect to cancer, there have been long-standing queries regarding the influence of PA on the risk of 
developing cancer; however, previous studies have shown that PA decreases the risk of breast cancer development in women and increases their 5-year survival rate $(14,15)$. In addition, it was demonstrated that DCs could induce an improved tumour-specific response in vitro following a short period of exercise (16). Furthermore, previous studies from our group demonstrated that moderate PA was able to potentiate the polarization of cluster of differentiation (CD) $4^{+}$T-cell and macrophage secretion profiles to anti-tumour patterns: Th1 and $\mathrm{M} 1$, respectively $(17,18)$.

Considering the immunosuppressive features of tumours during their development and progression (19), the authors of the present study hypothesise that prolonged PA may improve the anti-tumour activity of the immune system. Therefore, the present study aimed to investigate DC maturation in a mouse model of 7,12-dimethyl-benzanthracene (DMBA)-induced immunosuppression that was subjected to PA.

\section{Materials and methods}

Animals. A total of 56 adult female Balb/c mice (8-weeks-old) from the Institute for Research in Oncology were used. The mice were housed in groups in plastic cages under a light/dark cycle at $21 \pm 3^{\circ} \mathrm{C}$, with food and water available ad libitum. Following the experimental period, the mice were sacrificed with an overdose of $50 \mathrm{mg} / \mathrm{kg}$ ketamine and $15 \mathrm{mg} / \mathrm{kg}$ xylazine. This study was approved by the Federal University of Triângulo Mineiro (Uberaba, Brazil) Ethics Committee on the Use of Animals (registration no. 160).

Experimental groups and DMBA induction. The mice were divided into the following four groups ( $n=14$ per group): i) Non-DMBA/non-PA group (GI); ii) non-DMBA/PA group (GII); iii) DMBA/non-PA group (GIII); and iv) DMBA/PA group (GIV). In the DMBA groups, the mice received daily oral gavage with $1 \mathrm{mg} / \mathrm{ml}$ DMBA for 6 weeks. There was a gap of 16 weeks prior to the initiation of training to permit carcinogenic activity.

Training. The mice underwent swimming training for 5 days per week for 8 weeks. Following a 15-day period of adaptation in water, mice in the trained groups were placed in water for 45 min of swimming on each training day. The water was changed daily and the water temperature was maintained in the range of $30-35^{\circ} \mathrm{C}$.

Bone marrow collection and culturing. Following sacrifice, bone marrow was removed from the femur and tibia of the mice, immediately homogenized, washed three times in incomplete Iscove's Modified Dulbecco's Medium (IMDM), and centrifuged at $290 \times \mathrm{g}$ for $10 \mathrm{~min}$ at $4^{\circ} \mathrm{C}$. Following centrifugation, the cells were counted and re-suspended in complete IMDM.

Bone marrow cells re-suspended in complete IMDM were distributed in flat-bottom, 6-well plates at a concentration of $5 \times 10^{6}$ cells/well. After 2 days of culturing, the cells were stimulated with granulocyte-macrophage colony-stimulating factor and IL-4 for 5 days, after which they were stimulated with tumour necrosis factor- $\alpha(\mathrm{TNF}-\alpha)$ and tumour lysate. In addition, half of the plates were stimulated with $10 \mu \mathrm{g} / \mathrm{ml}$ lipopolysaccharide (LPS; Sigma-Aldrich; Merck Millipore, Darmstadt, Germany). After $48 \mathrm{~h}$ of incubation at $37^{\circ} \mathrm{C}$ in $5 \%$ $\mathrm{CO}_{2}$, supernatant samples were obtained, which were divided into six aliquots per incubation time point and stored at $-80^{\circ} \mathrm{C}$. The adherent cells were collected for flow cytometry.

Flow cytometry. Bone marrow-derived DCs (BMDCs) were immunolabelled with peridinin chlorophyll-protein complex cyanine 5.5-conjugated anti-CD11b antibody (rat anti-mouse monoclonal; 550993; undiluted; BD Biosciences, San Jose, CA, USA) to reveal total DCs, allophycocyanin (APC)-conjugated anti-CD11c antibody (hamster anti-mouse monoclonal; 553956; undiluted; BD Biosciences) to reveal mature DCs, and fluorescein isothiocyanate-conjugated anti-CD80 (hamster anti-mouse monoclonal; 553768; undiluted; BD Biosciences), APC-conjugated anti-CD86 (rat anti-mouse monoclonal; 558703; undiluted; BD Biosciences) and phycoerythrin-conjugated anti-I-A (rat anti-mouse monoclonal; 562010; undiluted; BD Biosciences) antibodies to detect the expression of costimulatory molecules. The cells were incubated with each antibody at $4^{\circ} \mathrm{C}$ for $30 \mathrm{~min}$ in the dark, and then washed with PBS to remove excess antibodies. Finally, cell aliquots were re-suspended in $500 \mu \mathrm{l}$ PBS for flow cytometric analysis using a BD FACSCalibur ${ }^{\mathrm{TM}}$ cytometer (BD Biosciences).

Cytokine levels. The levels of the cytokines interferon (IFN) $-\gamma$, IL-4, IL-12 and TGF- $\beta$ in the supernatant samples were measured using ELISAs with pairs of monoclonal antibodies purchased from BD OptEIA ${ }^{\mathrm{TM}}$ (BD Biosciences), according to the manufacturer's protocol. Results show the index between the supernatants with stimulus (LPS) and supernatants without stimulus. All cytokines were measured in $\mathrm{pg} / \mathrm{ml}$.

Statistical analysis. Data were assessed for normality of distribution. For variables in which the distribution was found to be normal, parametric tests were performed, and for those which the distribution was not normal, non-parametric tests were conducted. For comparisons of normally distributed data, Student's t-tests were performed for two-group comparisons and analysis of variance was used for comparisons among three or more groups. Normally distributed data are expressed as the mean \pm standard error of mean. For comparisons of non-normally distributed data, Mann-Whitney U tests were performed for two-group comparisons and Kruskal-Wallis and post-hoc Dunn's tests were used for comparisons among three or more groups. Non-normally distributed data are expressed as the median (range). $\mathrm{P}<0.05$ was considered to indicate a statistically significant difference.

\section{Results}

Characterization of DC phenotype according to the expression of costimulatory molecules. For the phenotypic characterization of the mouse BMDCs, the cells were labelled with antibodies against CD11b and CD11c, as well as MHC II and CD80 and CD86 costimulatory molecules, and analysed by flow cytometry. As shown in Fig. 1, DMBA did not exert a suppressive effect on the differentiation of DCs. The groups submitted to PA in the presence (GIV) or absence (GII) of DMBA showed significantly higher levels of $\mathrm{CD} 11 \mathrm{~b}^{+} / \mathrm{CD} 11 \mathrm{c}^{+}$ 

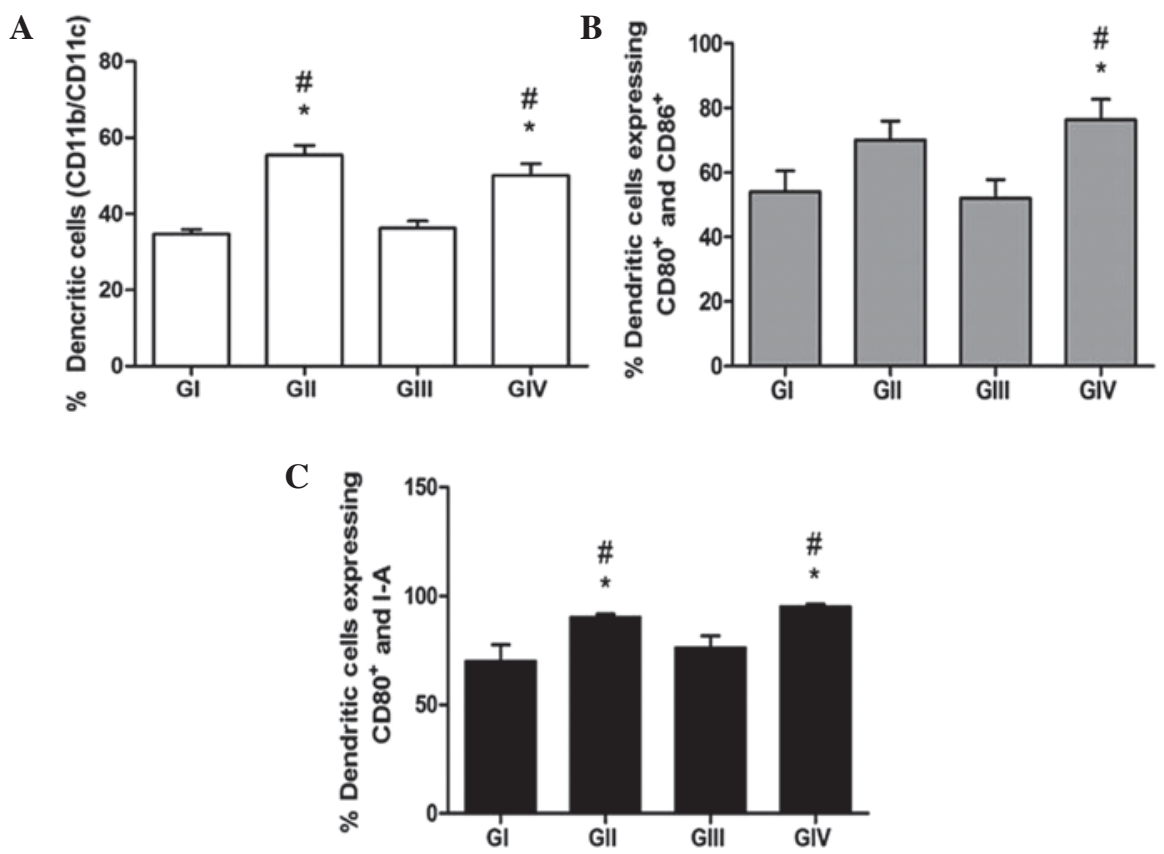

Figure 1. Immunolabelling of bone marrow-derived dendritic cells (BMDCs) from the various groups. (A) BMDCs were labelled with anti-CD11b and anti-CD11c antibodies to reveal total and mature DCs, respectively. (B) Co-expression of CD80 and CD86, and (C) co-expression of CD80 and major histocompatibility complex class II, were assessed by immunolabelling and flow cytometry. Data are presented as the mean \pm standard error of the mean. ${ }^{*} \mathrm{P}<0,05$ vs. GI. "P<0,05 vs. GIII. GI, non-7,12-dimethyl-benzanthracene (DMBA)/non-physical activity (PA) group; GII, non-DMBA/PA group; GIII, DMBA/non-PA group; GIV, DMBA/PA group; CD, cluster of differentiation.

DCs, as compared with GI and GIII mice $(\mathrm{P}<0.05)$. These results suggest a greater differentiation capacity for BMDCs in vitro following PA. Similarly, GII and GIV showed significantly higher expression levels of CD80, CD86 and MHC II, as compared with GI and GIII $(\mathrm{P}<0.05)$, thus suggesting that PA induces the differentiation and maturation of DCs, even within an immunosuppressive environment, as induced by DMBA.

Synthesis of cytokines by DCs. The cytokine profile of BMDCs was analysed using ELISAs. As is shown in Fig. 2, BMDCs from the GII and GIV mice showed higher synthesis levels of IL-12, as compared with those from the GI and GII mice. Furthermore, the secretion levels of IL-12 were markedly higher for the GIV mice compared with the GIII mice $(\mathrm{P}=0.063)$.

When analysing IFN- $\gamma$ synthesis, the groups that experienced PA (GII and GIV) showed higher expression levels, and a significant difference was observed for GIV compared with GI and GIII ( $\mathrm{P}<0.05$; Fig. 2).

Conversely, the synthesis levels of IL-4 showed an antagonistic behaviour to IL-12 and IFN- $\gamma$; they were significantly increased in GIII compared with the other groups $(\mathrm{P}<0.05)$.

There were no significant changes in the secretion of TGF- $\beta$ among the groups (P>0.05; Fig. 2D). However, GIII showed a discreet increase in the production of TGF- $\beta$, as compared with the other groups, and GIV showed attenuated expression of this cytokine.

\section{Discussion}

Physical activity has been shown to promote anti-tumour responses (20), and this effect may occur via the regulation of genes that have been implicated in cancer prevention (21), or through favourable effects on immune responses (20).

In our previous studies, we investigated the effect of PA on immunological behaviour, including T-cell and macrophage profiles $(17,18)$. It was demonstrated that PA induced a decrease in the number of regulatory T-cells (Treg) in animal spleens, and there was an increase in the number of $\mathrm{CD} 4^{+} \mathrm{T}$-cells expressing IFN- $\gamma$, IL-12 and TNF- $\alpha$, which are associated with a Th1 profile (17). Furthermore, the levels of IL-12 and IFN- $\gamma$, which are associated with an M1 macrophage profile, were increased under the influence of PA (18). Based on these findings, the objective of this study was to assess the influence of PA in the maturation process of BMDCs under immunosuppressive conditions, in order to improve our understanding of the behaviour of BMDCs under these conditions.

Although the clinical effects of PA under immunosuppressive conditions, such as in cancer patients, are not yet fully understood, the use of a prolonged exercise protocol in this study was justified, given the previously described benefits of PA. In the present study, female Balb/c mice served as experimental models and carcinogenesis was induced using DMBA, a traditional carcinogen that also has immunosuppressive properties $(17,18)$.

In the present study, mice that performed PA and received DMBA (GIV) showed external tumour masses, while mice that received DMBA but did not perform PA (GIII) had a frequency of external tumour masses of 35\%. Similar findings were observed in a study by Lane et al (22), wherein animals that performed PA and consumed a balanced diet had lower levels of tumours compared with animals that did not perform exercise. Furthermore, in a previous study, the body composition of animals that performed PA was not altered by the 
A

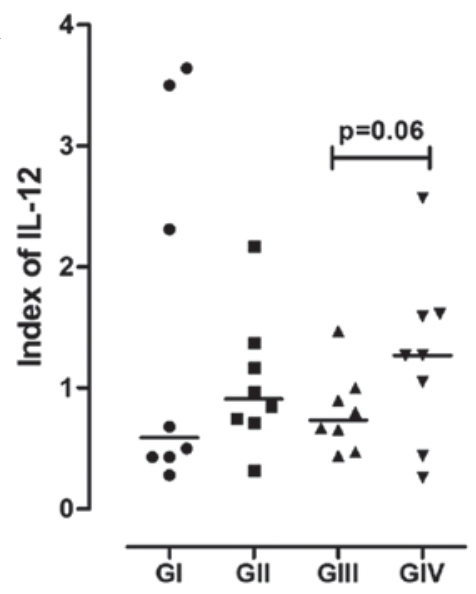

C

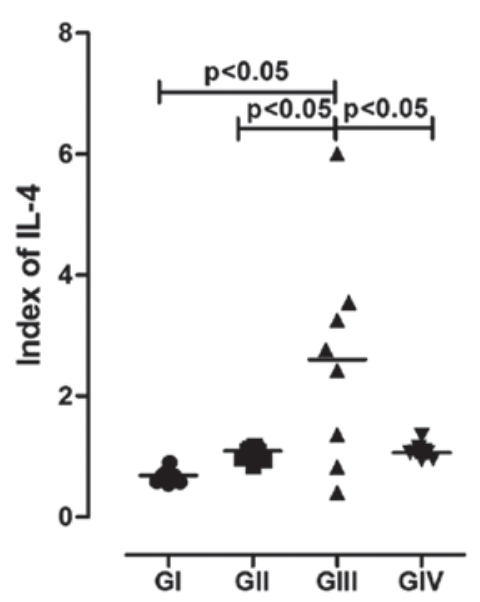

B

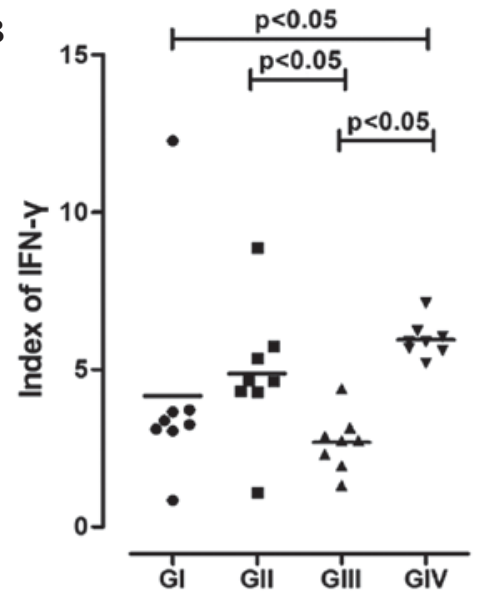

D

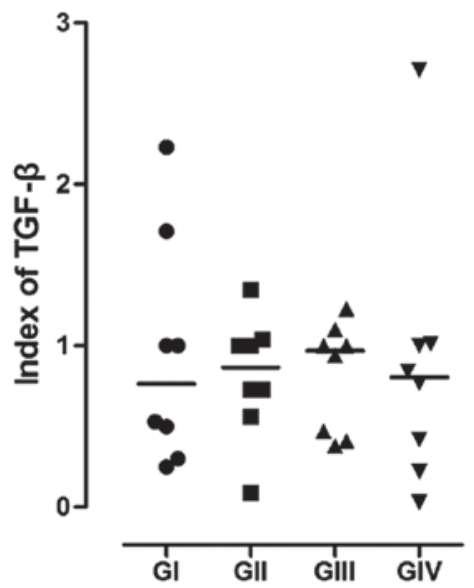

Figure 2. Representation of cytokines synthesised by bone marrow-derived dendritic cells (BMDCs). The levels of (A) IL-12, (B) IFN- $\gamma$, (C) IL-4 and (D) TGF- $\beta$ in the supernatant of BMDCs from the various groups were determined using ELISAs. GI, non-7,12-dimethyl-benzanthracene (DMBA)/non-physical activity (PA) group; GII, non-DMBA/PA group; GIII, DMBA/non-PA group; GIV, DMBA/PA group. IL, interleukin; IFN- $\gamma$, interferon- $\gamma$; TGF- $\beta$, transforming growth factor- $\beta$.

exercise regimen, although trained animals showed greater tumor masses compared with the sedentary group (23).

In order for an anti-tumour immune response to be effective, its various components must work synergistically; the components of the innate immune response, including macrophages, natural killer cells and DCs (3), must act together with those of the acquired immune response, including $\mathrm{T}$ and B lymphocytes (4).

Previous studies demonstrated that mature DCs were able to induce a potent and specific anti-tumour response by stimulating T-cells, in particular CD8 T-cells, and inhibiting metastasis to the lungs $(3,24)$.

In the present study, bone marrow cells were shown to differentiate into DCs that underwent maturation following culturing under the influence of stimulatory factors, in spite of the immunosuppressive effects of DMBA. BMDCs from GIII mice (DMBA induction without PA) showed a higher synthesis level of suppressive cytokines. However, the number of differentiated BMDCs was significantly higher in the groups that performed PA, and the expression pattern of cytokines was associated with Th1 and M1 profiles.

The expression levels of costimulatory molecules were also increased in BMDCs from the mice that were subjected to PA. These results were consistent with those reported in previous studies $(25,26)$, wherein PA was associated with an increase in the number of DCs, as well as increases in the expression of MHC class II, CD80 and CD86. However, Ru and Peijie (27) reported that over-training could induce immunosuppression by reducing the number of DCs and costimulatory molecule expression. Chiang et al (25) demonstrated that the groups subjected to PA produced higher levels of IL-12 compared with sedentary groups, mainly when comparing groups under DMBA induction (GIII vs. GIV). In the present study, the groups that underwent a training protocol showed increased expression levels of IL-12 and IFN- $\gamma$, and reduced expression levels of suppressive cytokines in the supernatant of cultured BMDCs.

In conclusion, the present study demonstrated that PA stimulated the maturation of BMDCs and increased the expression of IL-12 and IFN- $\gamma$, and decreased the synthesis of IL-4 and TGF- $\beta$, by these cells. These results suggested a potential polarization of the immune response towards a Th1 profile, which is thought to exert anti-tumour effects. Therefore, the authors of the present study suggest that a combination of DC-based immunotherapy and PA should be investigated in further studies, in particular with regard to the activation of tumour-specific T-cells by DCs and tumour-infiltration by immune cells. Furthermore, patients with cancer should consider performing regular PA to complement conventional therapies, including chemotherapy, radiotherapy and surgery, as well as newer therapies, such as immunotherapy. 


\section{Acknowledgements}

The present study was supported by grants from the Foundation for Research Assistance of the State of Minas Gerais (grant no. FAPEMIG-RED-00011-14), the National Council for Scientific and Technological Development (grant no. CAPES-PDSE 0592/13-7) and the Uberaba Foundation for Teaching and Research.

\section{References}

1. Ashley DM, Faiola B, Nair S, Hale LP, Bigner DD and Gilboa E: Bone marrow-generated dendritic cells pulsed with tumor extracts or tumor RNA induced antitumor immunity against central nervous system tumors. J Exp Med 186: 1177-1182, 1997.

2. Banchereau J and Steinman RM: Dendritic cells and the control of immunity. Nature 392: 245-252, 1998.

3. Fields RC, Shimizu K and Mulé JJ: Murine dendritic cells pulsed with whole tumor lysates mediate potent antitumor immune responses in vitro and in vivo. Proc Natl Acad Sci USA 95: 9482-9487, 1998.

4. Zitvogel LZ, Mayordomo JI, Tjandrawan T, DeLeo AB, Clarke MR, Lotze MT and Storkus WJ: Therapy of murine tumors with tumor peptidepulsed dendritic cells: Dependence on T cells, B7 costimulation, and T helper cell 1-associated cytokines. J Exp Med 183: 87-97, 1996.

5. Gabrilovich DI, Chen HL, Girgis KR, Cunningham HT, Meny GM, Nadaf S, Kavanaugh D and Carbone DP: Production of vascular endothelial growth factor by human tumors inhibits the functional maturation of dendritic cells. Nat Med 2: 1096-1103, 1996.

6. Gastl GA, Abrams JS, Nanus DM, Oosterkamp R, Silver J, Liu F, Chen M, Albino AP and Bander NH: Interleukin-10 production by human carcinoma cell lines and its relationship to interleukin-6 expression. Int J Cancer 55: 96-101, 1993.

7. Kavanaugh DY and Carbone DP: Immunologic dysfunction in cancer. Hematol Oncol Clin North Am 10: 927-951, 1996.

8. Wojtowicz-Praga S: Reversal of tumor-induced immunosuppression: A new approach to cancer therapy. J Immunother 20 : 165-177, 1997.

9. LaVoy EC, Bollard CM, Hanley PJ, O'Connor DP, Lowder TW, Bosch JA and Simpson RJ: A single bout of dynamic exercise by healthy adults enhances the generation of monocyte-derived-dendritic cells. Cell Immunol 295: 52-59, 2015.

10. Blannin AK, Gleeson M and Brooks S: Effect of lactacidosis on human leucocyte adherence: A possible explanation of why the leucocyte count continues to rise after cessation of very high intensity exercise. In: Gleeson, M. Immune function in sport and exercise. Philadelphia, Elsevier, 67-89, 2006.

11. Fairey AS, Courneya KS, Field CJ and Mackey JR: Physical exercise and immune system function in cancer survivors. Cancer 94: 539-551, 2002.
12. Woods JA: Physical activity, exercise, and immune function. Brain Behav Immun 19: 369-370, 2005.

13. Petersen AM and Pedersen BK: The anti-inflammation effect of exercise. J Appl Physiol (1985) 98: 1154-1162, 2005.

14. Cleveland RJ, Eng SM, Stevens J, Bradshaw PT, Teitelbaum SL, Neugut AI and Gammon MD: Influence of prediagnostic recreational physical activity on survival from breast câncer. Eur J Cancer Prev 21: 46-54, 2012.

15. Friedenreich CM and Rohan TE: Physical activity and risk of breast cancer. Eur J Cancer Prev 4: 145-151, 1995.

16. LaVoy EC, Bollard CM, Hanley PJ, O'Connor DP, Bosch JA and Simpson RJ: A single bout of dynamic exercise enhances the expansion of MAGE-A4 and PRAME-specific cytotoxic t-cells from healthy adults. Exerc Immunol Rev 21: 144-153, 2015.

17. Abdalla DR, Murta EF and Michelin MA: The influence of physical activity on the profile of immune response cells and cytokine synthesis in mice with experimental breast tumors induced by 7,12-dimethylbenzanthracene. Eur J Cancer Prev 22: 251-258, 2013.

18. Abdalla DR, Aleixo AA, Murta EF and Michelin MA: Innate immune response adaptation in mice subjected to administration of DMBA and physical activity. Oncol Lett 7: 886-890, 2014.

19. Terme M, Ullrich E, Aymeric L, Meinhardt K, Coudert JD, Desbois M, Ghiringhelli F, Viaud S, Ryffel B, Yagita H, et al: Cancer-induced immunosuppression: IL-18-elicited immunoablative NK cells. Cancer Res 72: 2757-2767, 2012.

20. Goh J, Endicott E and Ladiges WC: Pre-tumor exercise decreases breast cancer in old mice in a distance-dependent manner. Am J Cancer Res 4: 378-384, 2014.

21. Buehlmeyer K, Doering F, Daniel H, Kindermann B, Schulz T and Michna H: Alteration of gene expression in rat colon mucosa after exercise. Ann Anat 190: 71-80, 2008.

22. Lane HW, Keith RE, Strahan S and White MT: The effect of diet, exercise and 7,12-dimethylbenz(a)anthracene on food intake, body composition and carcass energy levels in female virgin BALB/c mice. J Nutr 121: 1876-1882, 1991.

23. Thompson HJ, Ronan AM, Ritacco KA, Tagliaferro AR and Meeker LD: Effect of exercise on the induction of mammary carcinogenesis. Cancer Res 48: 2720-2723, 1988.

24. Asavaroengchai W, Kotera Y and Mulé JJ: Tumor lysate-pulsed dendritic cells can elicit an effective antitumor immune response during early lymphoid recovery. Proc Natl Acad Sci USA 99: 931-936, 2002.

25. Chiang LM, Chen YJ, Chiang J, Lai LY, Chen YY and Liao HF: Modulation of dendritic cells by endurance training. Int J Sports Med 28: 798-803, 2007

26. Liao HF, Chiang LM, Yen CC, Chen YY, Zhuang RR, Lai LY, Chiang $\mathrm{J}$ and Chen YJ: Effect of a periodized exercise training and active recovery program on antitumor activity and development of dendritic cells. J Sports Med Phys Fitness 46: 307-314, 2006.

27. $\mathrm{Ru} \mathrm{W}$ and Peijie C: Modulation of dendritic cells and NKT cells excessive exercise in rats. J Med Biol Eng 29: 190-194, 2009. 